\title{
Distribution of Interstitial Stem Cells in Hydra
}

\author{
Charles N. David and Ida Plotnick \\ Department of Molecular Biology, Albert Einstein College of Medicine, Bronx, New York 10461
}

Received August 17, 1979; accepted in revised form October 29, 1979

\begin{abstract}
The distribution of interstitial stem cells along the Hydra body column was determined using a simplified cloning assay. The assay measures stem cells as clone-forming units (CFU) in aggregates of nitrogen mustard inactivated Hydra tissue. The concentration of stem cells in the gastric region was uniform at about $0.02 \mathrm{CFU} /$ epithelial cell. In both the hypostome and basal disk the concentration was 20 -fold lower. A decrease in the ratio of stem cells to committed nerve and nematocyte precursors was correlated with the decrease in stem cell concentration in both hypostome and basal disk. The ratio of stem cells to committed precursors is a sensitive indicator of the rate of self-renewal in the stem cell population. From the ratio it can be estimated that $<10 \%$ of stem cells self-renew in the hypostome and basal disk compared to $60 \%$ in the gastric region. Thus, the results provide an explanation for the observed depletion of stem cells in these regions. The results also suggest that differentiation and self-renewal compete for the same stem cell population.
\end{abstract}

\section{INTRODUCTION}

Interstitial stem cells in Hydra continuously give rise to differentiated nerve cells and nematocytes (see Bode and David, 1978 , for review). Per stem cell generation, $60 \%$ of stem cells divide to yield further stem cells, $30 \%$ initiate nematocyte differentiation, and $10 \%$ initiate nerve differentiation (David and Gierer, 1974). Under these conditions the stem cell population grows exponentially doubling every 3.5 days in parallel with the growth of $H y d r a$ tissue.

The growth rate of the stem cell population in Hydra is controlled by the fraction $\left(P_{\mathrm{s}}\right)$ of stem cell daughters that remain stem cells (self-renew) in each stem cell generation. Several experiments now indicate that the value of $P_{\mathrm{s}}$ depends on the density of stem cells in tissue. (1) Depletion of stem cells in Hydra increases the value of $P_{s}$ causing the stem cell population to grow more rapidly than the tissue as a whole and thus recover its normal density (Bode $e t$ $a l$., 1976). (2) Stem cells seeded at low densities in feeder layers manifest high $P_{s}$; stem cells seeded at high densities manifest low $P_{\mathrm{s}}$ (Sproull and David, 1979). (3) During the growth of stem cell clones the value of $P_{\mathrm{s}}$ decreases as the number of stem cells in the clone increases (David and MacWilliams, 1978). These observations all indicate that $P_{\mathrm{s}}$ is regulated by negative feedback from neighboring stem cells. A model has been proposed to explain this behavior. Stem cells are postulated to secrete a factor which diffuses into the environment around the cells; stem cells are sensitive to the factor and use the ambient factor concentration to regulate the value of $P_{s}$ (David and MacWilliams, 1978). According to the model, low stem cell concentrations lead to low factor concentrations and high values of $P_{\mathrm{s}}$; high stem cell concentrations lead to high factor concentrations and low values of $P_{s}$. This negative feedback loop maintains the stem cell population at a constant density as is indeed observed in Hydra tissue (Bode et al., 1977).

The model predicts that the concentration of stem cells over time should be uniform in all parts of Hydra and, furthermore, that stem cells should occupy all available interstitial space in Hydra. The distribution 
of stem cells in Hydra is not presently known. However, the distribution of interstitial cells, of which the stem cells are a subpopulation, is already known to be nonuniform. Direct cell counts indicate that interstitial cells are less abundant in both hypostome and basal disk compared to the gastric region (Bode et al., 1973; David and Challoner, 1974). Stained preparations of whole animals confirm this result and demonstrate that the interstitial cell population has a sharp upper boundary at a position just below the tentacle ring and a sharp lower boundary just above the basal disk (David, 1975).

Neither of the above results yields information about the distribution of stem cells specifically, and it is possible that the actual stem cell distribution is significantly different from that of the interstitial cell population as a whole. In the present report we have determined the distribution of stem cells in Hydra with the intent of understanding its relationship to the model for the control of stem cell self-renewal. We present results on the distribution of stem cells in all regions of $H y d r a$. We have assayed stem cells using a modification of the clonal assay (David and Murphy, 1977) in which feeder layers are inoculated with large numbers of stem cells and the total size of the stem cell population determined after a defined period of growth. This modification simplifies and increases the accuracy of the clonal assay. We have, in addition, determined the ratio of stem cells to early committed cells in each region of $\mathrm{Hy}$ $d r a$. This ratio is a sensitive indicator of the value of $P_{\mathrm{s}}$ and we have used it to estimate the growth rate of the stem cell population in specific regions of Hydra.

The results indicate that stem cells are uniformly distributed along the body column in the gastric region but are almost absent in the hypostome and basal disk. The low density of stem cells in hypostome and basal disk is correlated with a decrease in the ratio of stem cells to carly committed cells. From this finding we estimate that $P_{\mathrm{s}}$ is $<0.1$ in the hypostome and basal disk compared to 0.6 in the gastric region. This local decrease in $P_{\text {s }}$ prevents growth of the stem cell population in the hypostome and basal disk thereby explaining the absence of stem cells from these regions. We discuss the possibility that nerve differentiation localized in the hypostome and basal disk is responsible for the low value of $P_{\mathrm{s}}$ in these regions.

\section{MATERIALS AND METHODS}

Hydra attenuata were cultured at $19^{\circ} \mathrm{C}$ in $10^{-3} \mathrm{M} \mathrm{CaCl}_{2}, 10^{-3} \mathrm{M} \mathrm{NaHCO}_{3}$, and $10^{-5}$ $M$ EDTA. Cultures were fed daily with Artemia nauplii. Six hours after feeding cultures were washed.

Large animals with two buds were used for the experiments described below. Buds were removed and the body column was cut into four sections. Cuts were made just below the tentacle ring and through the middle of the peduncle; the remaining body column section was divided into two equal pieces. The average size of each piece in terms of number of epithelial cells is: hypostome: 3900 , upper body column: 7000 , lower body column: 8100 , and basal disk: 2000. The total number of cells in such Hydra is about 65,000 , of which about 4800 are $1 \mathrm{~s}+2 \mathrm{~s}$ (see below).

Identification of stem cells and early committed cells by maceration. The maceration technique (David, 1973) permits identification and quantitation of all Hydra cell types including epithelial cells, interstitial cells, differentiating nematoblasts, and differentiated nerves and nematocytes. Maceration dissociates Hydra tissue into single cells or small clusters in the case of interstitial cells held together by cytoplasmic bridges; cells retain their in vivo morphology in macerations enabling easy identification by phase contrast microscopy. Stem cells and early committed precursors to nerve and nematocyte differentiation occur in macerations as large single cells and 
in clusters of two. We refer to this class of cells as 1s+2s (David and MacWilliams, 1978; Sproull and David, 1979). Epithelial cells make up the ectodermal and endodermal epithelium of Hydra; the number of epithelial cells is a convenient measure of epithelial surface area. The ratio of $1 \mathrm{~s}+2 \mathrm{~s}$ to epithelial cells is thus a measure of the concentration of $1 \mathrm{~s}+2 \mathrm{~s}$ in Hydra tissue (Bode et al., 1973).

Because of the low numbers of cells in many of the samples reported here, macerations were prepared and analyzed using the modified procedure described by Sproull and David (1979).

Growth of interstitial stem cells in nitrogen mustard (NM) aggregates. Interstitial stem cells were cultured in aggregates of nitrogen mustard-treated Hydra tissue (David and Murphy, 1977; Sproull and David, 1979). The aggregates function as feeder layers. To prepare stem cell cultures, NM host tissue and live tissue were each dissociated in cell culture medium (Gierer et $a l ., 1972$ ). Aliquots of live cell suspension containing from 1-100 stem cells were mixed with NM cell suspension and the mixture was centrifuged to form a pellet. Cell pellets were removed from the tubes and incubated as previously described (David and Murphy, 1977).

Assay of clone-forming units (CFU). NM aggregates containing an average of $1 \mathrm{CFU}$ per aggregate were prepared, incubated for 10 days, stained with toluidine blue, and examined as whole mounts (David and Murphy, 1977). Stem cell clones were identified as patches of darkly staining interstitial cells in the ectoderm of the aggregates. Clones are derived from single stem cells (David and Murphy, 1977) and the number of clones formed by a live cell suspension is a measure of clone-forming units (CFU) in the suspension. The titer of CFU in a live cell suspension is about $13 \%$ of the stem cell titer due to cell losses in the aggregation procedure and a stem cell cloning efficiency less than one (David and Murphy, 1977).
Rapid assay for $C F U$. A new rapid assay for CFU is described in the results section.

Computer simulations. Computer simulations of stem cell growth and differentiation have been described in detail previously (Sproull and David, 1979).

Toluidine blue staining. Whole mounts of Hydra were prepared and stained as described by Diehl and Burnett (1964).

\section{RESULTS}

\section{Rapid Assay for Interstitial Stem Cells}

Interstitial stem cells can be assayed as the concentration of cells capable of forming clones in NM aggregates (David and Murphy, 1977). This assay is cumbersome since accurate scoring of clones requires only one to two clones per aggregate and 10-14 day incubation times to permit clones to grow to large size for scoring. These facts restrict the usefulness of the assay. We describe here an alternative assay which circumvents these problems. Recently Sproull and David (1979) have shown that the growth rate of stem cells in NM aggregates is independent of the number of CFU seeded in aggregates up to about $100 \mathrm{CFU}$ per aggregate. It thus appeared possible to assay stem cells by inoculating large numbers of stem cells into NM aggregates and scoring the number of stem cells in the entire aggregate following a defined period of growth. We chose 4 days as the period of growth because the only cells occurring as $1 s+2 s$ in aggregates at this time are in clones derived from stem cells inoculated on Day 0. (Differentiation intermediates which are seeded in NM aggregates along with stem cells have completed differentiation as nerves and nematocytes by Day 4.) The number of $1 \mathrm{~s}+2 \mathrm{~s}$ per aggregate on Day 4 , therefore, can be used to estimate the number of stem cells inoculated in the aggregate.

Figure 1 shows the results of several experiments in which aggregates were seeded with increasing amounts of a suspension of dissociated cells, incubated for 4 days, 


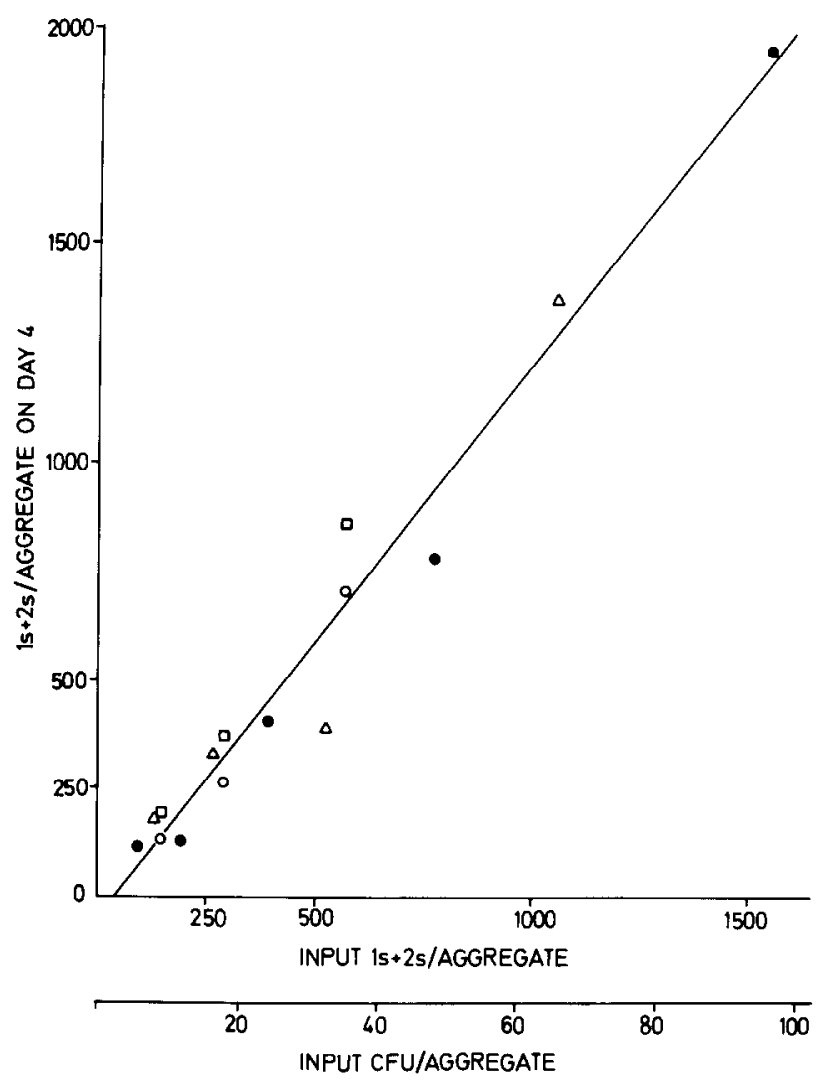

FIG. 1. Growth of interstitial stem cells in NM aggregates. NM aggregates were inoculated with increasing amounts of a live cell suspension, incubated for 4 days, macerated, and scored for $1 \mathrm{~s}+2 \mathrm{~s}$. The concentration of $1 \mathrm{~s}+2 \mathrm{~s}$ and $\mathrm{CFU}$ in the input cell suspension was determined on parallel samples. Different symbols indicate the results of four independent experiments. A least squares regression line has been drawn through the experimental values.

macerated, and scored for $1 \mathrm{~s}+2 \mathrm{~s}$. The results show that the number of $1 \mathrm{~s}+2 \mathrm{~s}$ per aggregate increases linearly with increasing size of the inoculum. To determine the titer of CFU in the live cell suspensions used in Fig. 1, samples of the suspensions were diluted and inoculated into NM aggregates at concentrations corresponding to about 1 CFU per aggregate. After 10 days of incubation, the aggregates were stained with toluidine blue and scored for clones (David and Murphy, 1977). The average of two determinations was $0.062 \mathrm{CFU} / 1 \mathrm{~s}+2 \mathrm{~s}$ in the input live cell suspension which is similar to previous estimates. This average value was used to construct a second abscissa in Fig. 1. From the slope of the curve, the ratio of $1 \mathrm{~s}+2 \mathrm{~s}$ per aggregate on Day 4 to input CFU is calculated as 19 .

The results above form the basis for a simplified assay for interstitial stem cells. Aliquots of live cell suspensions containing 1-100 CFU are inoculated into NM aggregates, incubated for 4 days, and the number of $1 \mathrm{~s}+2 \mathrm{~s} /$ aggregate is determined. When divided by 19 , this number yields the titer of CFU in the inoculum.

\section{Distribution of Interstitial Cells along Body Column}

To determine the distribution of interstitial cells along the body column, large $H y$ $d r a$ were cut into four sections: hypostome, upper gastric, lower gastric, and basal disk. The budding region is included in the lower gastric section. The peduncle was divided 
equally between the lower gastric section and the basal disk section. The cuts for hypostome and basal disk were made through the position at which the boundary to the interstitial cell population is usually located (Bode et al., 1973; David and Challoner, 1974; David, 1975). The sections were macerated and the number of $1 \mathrm{~s}+2 \mathrm{~s}$ and epithelial cells per section was determined. The results for three independent experiments are shown in Fig. 2A. The concentrations of $1 \mathrm{~s}+2 \mathrm{~s}$ in the upper and lower body column sections are essentially constant at about $0.3 \mathrm{ls}+2 \mathrm{~s} /$ epithelial cell. The concentration of $1 \mathrm{~s}+2 \mathrm{~s}$ is 8 - to 20 -fold lower in the hypostome and 5- to 10-fold lower in the basal disk.

The sharp boundary to the interstitial cell population in the hypostome and basal disk can be clearly visualized in whole mounts of Hydra stained with toluidine blue (Fig. 3). Toluidine blue stains interstitial cells and differentiating nematoblasts strongly; epithelial cells stain weakly and do not interfere with the visualization of interstitial cells. Fig. 3b shows a typical portion of the gastric region where interstitial cells and nematoblasts essentially saturate the spaces between epithelial cells. Figs. 3a and c show hypostome and basal disk, respectively. The ectoderm of both regions is essentially empty except for rare, isolated interstitial cells and nests of differentiating nematoblasts. The empty zone extends proximally from the hypostome a short distance down the body column and distally from the basal disk up the body column to a position in the middle of the peduncle. The boundary between the empty zone and the interstitial cell population in the body column is very sharp extending over a distance of only 5-10 cell diameters. Because the boundary region is sharp and slightly variable in position from animal to animal, we interpret the variations between experiments in the concentration of $1 \mathrm{~s}+2 \mathrm{~s}$ in these regions (Fig. 2A) as due to errors in cutting along the narrow and invisible (in living animals) boundary region.

\section{Distribution of Stem Cells along the Body Column}

The concentration of CFU was determined in tissue sections cut simultaneously
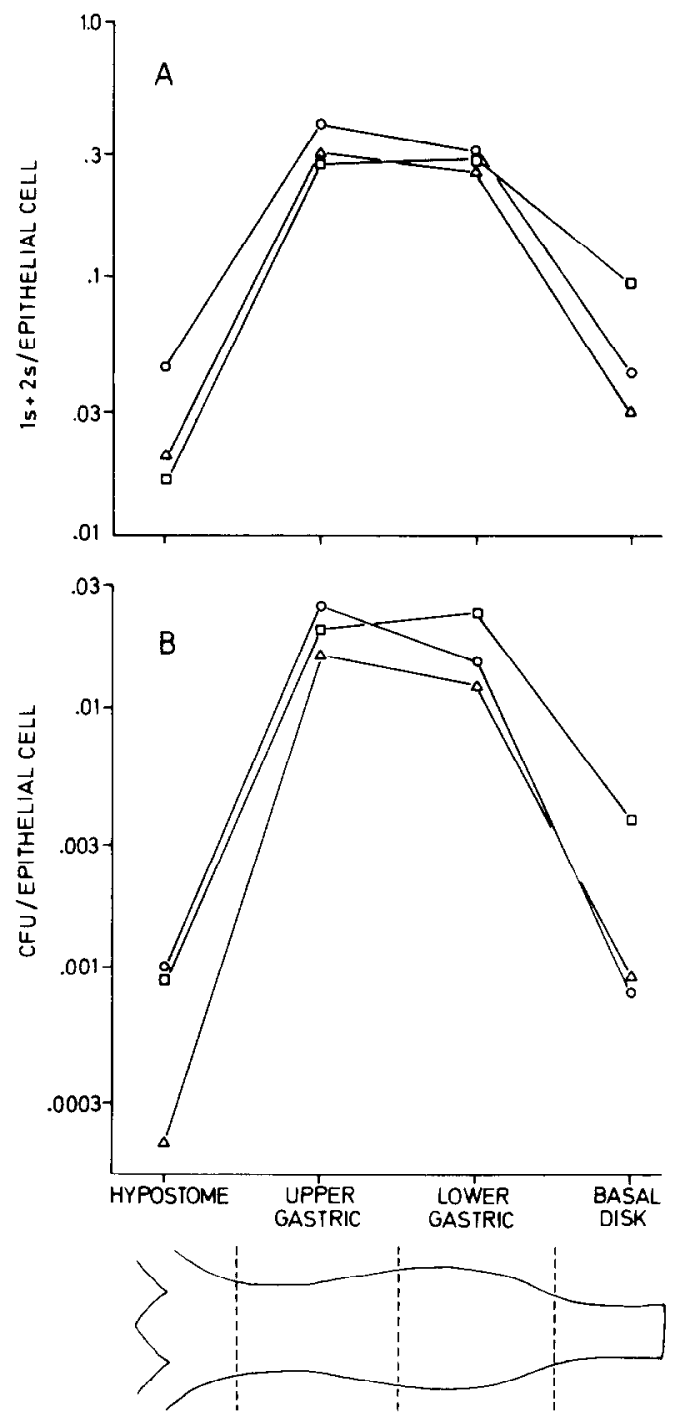

FIG. 2. Distribution of $1 \mathrm{~s}+2 \mathrm{~s}(\mathrm{~A})$ and $\mathrm{CFU}$ (B) along the Hydra body column. $1 \mathrm{~s}+2 \mathrm{~s}$ and epithelial cells were determined by maceration using pieces cut from 10 Hydra. CFU were determined on pieces cut from 20 Hydra using the rapid assay described under Results. Different symbols represent results from independent experiments. Note that basal disk section includes the proximal half of the peduncle. 

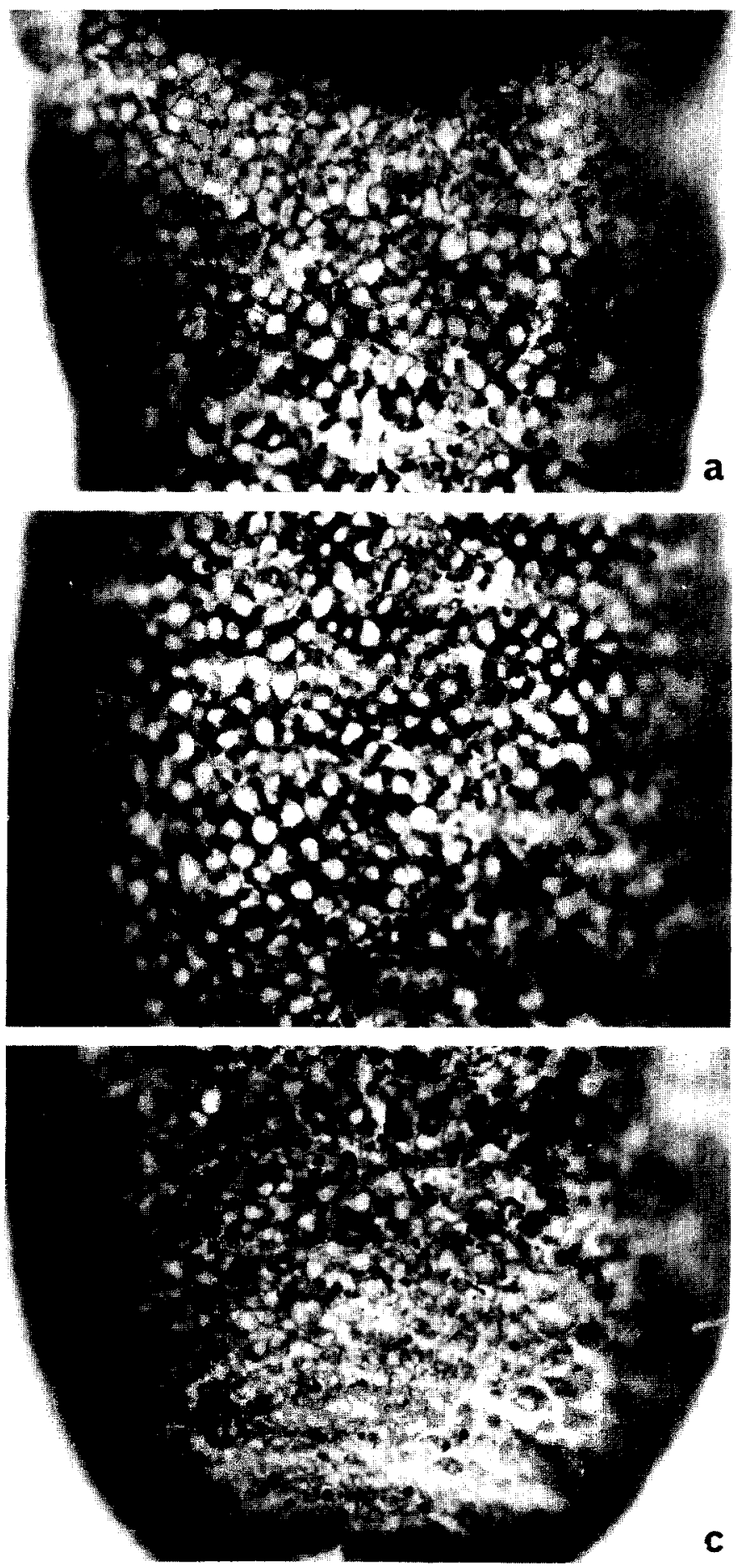

FIG. 3. Toluidine blue-stained whole mount of Hydra. Microscope is focused on the cetoderm. Interstitial cells and differentiating nematoblasts are the darkly staining clusters of cells. (a) Distal one-tenth of body column just below tentacle ring. (b) Portion of the gastric region. (c) Basal disk and proximal one-fifth of body column. $\times 57$. 
with those for the determination of $1 \mathrm{~s}+2 \mathrm{~s}$. The sections were dissociated and aliquots of dissociated cells seeded in NM aggregates. Aggregates were macerated after 4 days incubation and the number of $1 \mathrm{~s}+2 \mathrm{~s}$ per aggregate was determined. The number of CFU was determined by dividing 1s+2s/aggregate by 19 (Fig. 1). The results of three independent experiments are shown in Fig. 2B. The concentration of CFU in upper and lower body column is approximately constant at about $0.02 \mathrm{CFU} /$ epithelial cell. The concentration of CFU decreases 20 - to 50-fold in the hypostome and 7 - to 15-fold in the basal disk compared to the body column. As observed with $1 s+2 s$, there is significant variation between experiments in the concentration of CFU in the hypostome and basal disk pieces. The variation is probably due to cutting since the boundary is so sharp that any small error in cutting in one piece can bring in more stem cells than are contributed by the other 20 pieces used in the assay.

\section{Ratio of Stem Cells to Early Committed Cells along Hydra Body Column}

The population of $1 \mathrm{~s}+2 \mathrm{~s}$ in Hydra is a mixture of stem cells and early committed precursors to nerve and nematocyte differentiation (David and Gierer, 1974; David and MacWilliams, 1978). The proportions of stem cells and early committed cells vary depending on the rate of stem cell self-renewal and on the ratio of nerve to nematocyte differentiation. In general, for a given ratio of nerve to nematocyte differentiation the proportion of stem cells among $1 s+2 s$ decreases with decreasing self-renewal (see Discussion). From the results in Figs. $2 \mathrm{~A}$ and $\mathrm{B}$ we have calculated the ratio of $\mathrm{CFU}$ to $1 \mathrm{~s}+2 \mathrm{~s}$ for each experiment. These results are plotted in Fig. 4 along with the results of one other experiment not shown in Fig. 2. The results in Fig. 4 demonstrate that the ratio of CFU/ $1 s+2 s$ is constant in both upper and lower gastric region but decreases about twofold in both hypostome and basal disk.

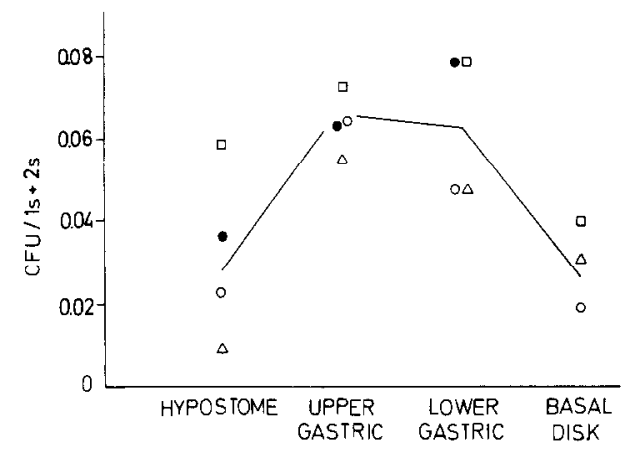

FIG. 4. Ratio of CFU $/ 1 \mathrm{~s}+2 \mathrm{~s}$ along the Hydra body column. Values are calculated from the results in Figs. $2 \mathrm{~A}$ and B. Symbols correspond to those in Fig. 2. Results of one additional experiment are also shown. Note that basal disk section includes the proximal half of the peduncle.

\section{DISCUSSION}

\section{Distribution of Stem Cells in Hydra}

The present experiments have made use of a new, rapid assay technique to determine the distribution of interstitial stem cells (CFU) along the body column of $\mathrm{Hy}$ dra. The concentration of stem cells throughout the gastric region is quite uniform at about $0.02 \mathrm{CFU} /$ epithelial cell (Fig. 2B). In both hypostome and basal disk there is a marked 20 -fold decrease in stem cell concentration to a level of about 0.001 CFU/epithelial cell (Fig. 2B). These results correspond to a total of about $300 \mathrm{CFU}$ (2300 stem cells; see under Materials and Methods) in the gastric region and about one to four CFU (8-30 stem cells) in the hypostome and basal disk.

\section{Estimates of $P_{s}$ in Hypostome, Gastric Re- gion, and Basal Disk}

The population of $1 s+2 s$ is a mixture of stem cells and early committed precursors to nerve and nematocyte differentiation. The relative proportion of each cell type depends on the value of $P_{\mathrm{s}}$ and the ratio of nematocyte to nerve differentiation. To calculate the proportions we have programmed a computer to simulate stem cell self-renewal, nerve differentiation, and nematocyte differentiation (Sproull and David, 1979). The flow of stem cells to each 
pathway can be independently varied and the number of stem cells and differentiating cells determined for each set of conditions. Figure 5 shows the ratio of stem cells $/ 1 \mathrm{~s}+2 \mathrm{~s}$ (CFU/1s+2s are shown on the second ordinate) as a function of $P_{\mathrm{s}}$ for varying ratios of nerve and nematocyte differentiation. The proportion of stem cells among $1 \mathrm{~s}+2 \mathrm{~s}$ decreases as $P_{\mathrm{s}}$ decreases. The quantitative course of the decrease depends on the ratio of nerve to nematocyte differentiation. $\mathrm{Ne}$ matocyte differentiation contributes more early committed cells to the pool of $1 s+2 s$ and therefore high proportions of nematocyte differentiation depress the $\mathrm{CFU} / 1 \mathrm{~s}+2 \mathrm{~s}$ ratio more than high proportions of nerve differentiation.

We can use the results in Fig. 4 and the simulations in Fig. 5 to estimate the value of $P_{\mathrm{s}}$ along the Hydra body column. Using a ratio of nematocyte to nerve commitment in the gastric region of 9:1 (David and Gierer, 1974; David, 1975) the CFU/1s+2s ratio of 0.062 (Fig. 4) suggests that $P_{\mathrm{s}}$ in this region is about 0.64 . This result is in good agreement with previous estimates (David and Gierer, 1974). In the hypostome and basal disk nerve differentiation occurs exclusively (David and Gierer, 1974). Under

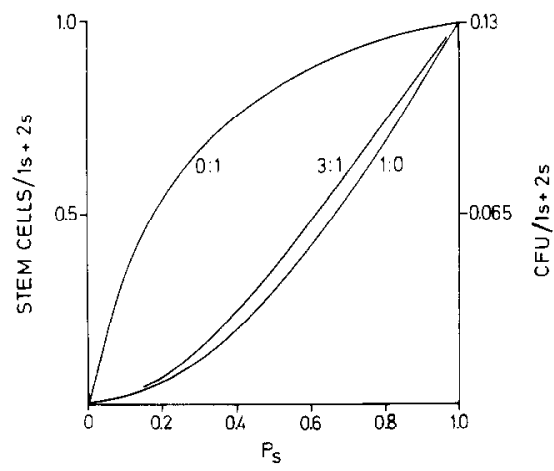

FIG. 5. Computer simulations of the ratio of stem cells $/ 1 \mathrm{~s}+2 \mathrm{~s}$ as a function of changing $P_{\mathrm{s}}$. The ratio of nematocyte:nerve commitment is given beside each curve. The $\mathrm{CFU} / 1 \mathrm{~s}+2 \mathrm{~s}$ ordinate was calculated using the observed value of $0.062 \mathrm{CFU} / 1 \mathrm{~s}+2 \mathrm{~s}$ (see under Results) for cells derived from whole Hydra in which $P_{\mathrm{a}}=0.6$ and the ratio of nematocyte:nerve commitment was 3:1 (David and Gierer, 1974). these conditions the observed CFU/1s+2s ratio of 0.03 suggests that $P_{\mathrm{s}}$ in these regions is less than 0.1 , indicating that these regions support virtually no stem cell proliferation. The results in Fig. 4 thus strongly support the conclusion that stem cell proliferation is inhibited locally in hypostome and basal disk and that this inhibition is the explanation for the very low concentration of stem cells in these regions.

An alternative explanation to the one above must also be considered. Interstitial cells have been shown to migrate along the body column of Hydra. The numbers of migrating cells are not large but there is a tendency for such cells to migrate apically (Herlands and Bode, 1974; Yaross and Bode, 1978). If migrating cells are uncommitted stem cells, then their migration into the hypostome would tend to increase the $\mathrm{CFU} / 1 \mathrm{~s}+2 \mathrm{~s}$ ratio and lead to overestimates of $P_{\mathrm{s}}$. Since $P_{\mathrm{s}}$ estimates are already very low, this would not affect the conclusion above. If, however, migrating cells were primarily committed nerve precursors their presence in these regions would tend to decrease the $\mathrm{CFU} / 1 \mathrm{~s}+2 \mathrm{~s}$ ratio and cause underestimates of $P_{\mathrm{s}}$. Under such circumstances the conclusion that $P_{\mathrm{s}}$ is low in these regions might be incorrect.

It is not presently known whether migrating cells are stem cells or committed nerve precursors or both. Nevertheless, it appears unlikely that selective migration of nerve precursors into the hypostome is responsible for the results in Fig. 4 since we have observed that isolation of the hypostome for $24 \mathrm{hr}$ before assaying does not change the ratio of CFU/1s+2s observed. Since isolation removes the hypostome from the source of migrating cells, a change in the values would have been expected if migrating nerve precursors contributed significantly to the population of $1 \mathrm{~s}+2 \mathrm{~s}$ in the hypostome. Thus the observed decrease in the $\mathrm{CFU} / 1 \mathrm{~s}+2 \mathrm{~s}$ ratio in the hypostome and basal disk must reflect a localized decrease in self-renewal. 


\section{Tissue Morphogenesis and the Control of $P_{s}$}

It is of interest to relate the present findings to previous work on the dynamics of the stem cell population in normal animals. Previous work has shown that the rate of self-renewal of the stem cell population in Hydra is controlled by the concentration of stem cells in tissue (Bode et al., 1976; David and MacWilliams, 1978; Sproull and David, 1979). Low stem cell concentrations cause high values of $P_{\mathrm{s}}$; high stem cell concentrations cause low values of $P_{\mathrm{s}}$. These results can be interpreted in terms of a model in which the value of $P_{\mathrm{s}}$ is regulated by negative feedback from neighboring stem cells. Additional evidence suggests that the feedback signal is mediated by a short-range diffusible factor.

Such a model predicts that the concentration of stem cells should be uniform in Hydra and that all available ectodermal space should be filled with stem cells. Irregularities in stem cell concentration or empty areas would tend to be evened out by local changes in $P_{\text {s. }}$. This prediction appears to be fulfilled for the stem cell population in the gastric region (Fig. 2B). However, the sharply lower stem cell concentration in both hypostome and basal disk is not consistent with the model and suggests that other factors influence the value of $P_{\mathrm{s}}$ in these regions.

To understand the nature of these factors it is necessary to review tissue movements in Hydra. Growth of Hydra tissue occurs uniformly throughout the hody column (Campbell, 1967; David and Campbell, 1972). Individual animals, however, do not increase in size since growth is just balanced by loss of tissue in the form of buds in the lower gastric region and by sloughing of tissue at the ends of the tentacles and from the basal disk. This combination of uniform growth and local cell loss leads to continuous movement of tissue either up the body column into the tentacles or down the body column into buds and basal disk. In the upper gastric region there is a region of nonmovement.

The stem cell population is embedded in the ectodermal epithelium. As the epithelium expands, the stem cell population expands with it such that the ratio of stem cells to epithelial cells remains constant. The stem cell population is also carried along by continuing movements of the epithelium. In the hypostome and basal disk, however, the coordinate expansion of stem cell and epithelial cell populations ceases since the ectoderm in these regions is empty of stem cells. The present experiments suggest that the explanation for this behavior is the low value of $P_{\mathrm{s}}$ in these regions. Since previous work has shown that nerve differentiation is concentrated in these same regions (David and Gierer, 1974), the simplest model is to assume that nerve differentiation localized in hypostome and basal disk is responsible for the low value of $P_{\mathrm{s}}$. Thus stem cells carried into the hypostome and basal disk by tissue movements are forced to differentiate as nerve cells, thereby emptying the epithelium of stem cells. Several independent experiments indicate that the localization of nerve differentiation in hypostome and basal disk is controlled by morphogenetic signals localized in these regions (see David, 1975; Bode and David, 1978, for review).

An essential feature of the simple model presented above is that the signals controlling nerve differentiation and self-renewal act on the same stem cell population. Without this assumption, it is not possible for nerve differentiation localized in the hypostome and basal disk to eliminate stem cells carried into these regions by tissue movements. Alternative models in which stem cells decisions are made in sequence: first self-renewal and then nerve versus nematocyte differentiation are not excluded by the results. However, in order to explain the observed distribution of stem cells and $P_{\mathrm{s}}$ in Hydra, such models require the as- 
sumption that stem cell position affects the value of $P_{\mathrm{s}}$ directly as well as the ratio of nerve to nematocyte differentiation.

This research was supported by grants from the NIH (GM 11301) and the NSF (77-25426). C.N.D. is recipient of a Career Development Award (FRA-132) from the American Cancer Society.

\section{REFERENCES}

Bode, H., Berking, S., David, C. N., Gierer, A., Schaller, H., and Trenkner, E. (1973). Quantitative analysis of cell types during growth and morphogenesis in Hydra. Wilhelm Roux Arch. Entwicklungsmech. Organismen 171, 269-285.

Bode, H., and David, C. N. (1978). Regulation of a multipotent stem cell, the interstitial cell of $H y d r a$. Prog. Biophys. Mol. Biol. 33, 189-206.

Bode, H., Flick, K., and Bode, P. (1977). Constraints on the relative sizes of the cell populations in Hydra attenuata. J. Cell Sci. 24, 31-50.

Bode, H. G., Flick, K. M., and Smith, G. S. (1976). Regulation of interstitial cell differentiation in $\mathrm{Hy}$ dra attenuata. I. Homeostatic control of interstitial cell population size. J. Cell Sci. 20, 29-46.

CAMPBELl, R. D. (1967). Tissue dynamics of steady state growth in Hydra littoralis. II. Patterns of tissue movement. J. Morphol. 121, 19-28.

David, C. N. (1973). A quantitative method for maceration of Hydra tissue. Wilhelm Roux Arch. Entwicklungsmech. Organismen 171, 259-268.

David, C. N. (1975). Stem cell differentiation in $\mathrm{Hy}$ dra. In "Microbiology 1975," pp. 435-441. Amer. Soc. for Microbiol., Boston.

David, C. N., and Campbell, R. (1972). Cell cycle kinetics and development of Hydra attenuata. I. Epithelial cells. J. Cell Sci. 11, 557-568.

David, C. N., and Challoner, D. (1974). Distribution of interstitial cells and differentiating nematocytes in nests in Hydra attenuata. Amer. Zool. 14, 537542.

David, C. N., and Gierer, A. (1974). Cell cycle kinetics and development of Hydra attenuata. III. Nerve and nematocyte differentiation. J. Cell Sci. 16, 359375.

David, C. N., and MacWilliams, H. (1978). Regulation of the self-renewal probability in Hydra stem cell clones. Proc. Nat. Acad. Sci. USA 75, 886-890.

David, C. N., and MurPHy, S. (1977). Characterization of interstitial stem cells in Hydra by cloning. Develop. Biol. 58, 372-383.

Diehl, F., and Burnett, A. L. (1964). The role of interstitial cells in the maintenance of Hydra. I. Specific destruction of interstitial cells in normal, sexual and non-budding animals. J. Exp. Zool. 155, 253-259.

Gierer, A., Berking, A., Bode, H., David, C. N., Flick, K., Hansmann, G., Schaller, C., and Trenkner, E. (1972). Regeneration of Hydra from reaggregated cells. Nature New Biol. 239, 98-101.

Herlands, R., and Bode, H. (1974). Oriented migration of interstitial cells and nematocytes in Hydra attenuata. Wilhelm Roux Arch. Entwicklungsmech. Organismen 176, 67-88.

Sproull, F., and David, C. N. (1979). Stem cell growth and differentiation in Hydra attenuata. I. Regulation of the self-renewal probability in multiclone aggregates. J. Cell Sci. 38, 155-169.

YAROSS, M., and BoDE, H. (1978). Regulation of interstitial cell differentiation in Hydra attenuata. IV. Nerve cell commitment in head regeneration is position-dependent. J. Cell Sci. 34, 27-38. 